\title{
Emerging patterns of New Physics with and without Lepton Flavour Universal contributions
}

\author{
Marcel Algueró ${ }^{1,2}$, Bernat Capdevila ${ }^{1,2, a}$, Andreas Crivellin ${ }^{3,4}$, Sébastien Descotes-Genon ${ }^{5}$, Pere Masjuan $^{1,2}$, \\ Joaquim Matias ${ }^{1,2}$, Javier Virto ${ }^{6,7}$ \\ ${ }^{1}$ Grup de Física Teòrica (Departament de Física), Universitat Autònoma de Barcelona, 08193 Bellaterra, Barcelona, Spain \\ ${ }^{2}$ Institut de Física d'Altes Energies (IFAE), The Barcelona Institute of Science and Technology, Campus UAB, 08193 Bellaterra, \\ Barcelona, Spain \\ ${ }^{3}$ Paul Scherrer Institut, CH-5232 Villigen PSI, Switzerland \\ ${ }^{4}$ Physik-Institut, Universität Zürich, Winterthurerstrasse 190, CH-8057 Zurich, Switzerland \\ ${ }^{5}$ Laboratoire de Physique Théorique, UMR 8627, CNRS, Univ. Paris-Sud, Université Paris-Saclay, 91405 Orsay Cedex, France \\ ${ }^{6}$ Physics Department T31, Technische Universität München, James Franck-Strasse 1, 85748 Garching, Germany \\ ${ }^{7}$ Center for Theoretical Physics, Massachusetts Institute of Technology, 77 Massachusetts Av., Cambridge, MA 02139, USA
}

Received: 27 May 2019 / Accepted: 31 July 2019 / Published online: 24 August 2019

(C) The Author(s) 2019

\begin{abstract}
We perform a model-independent global fit to $b \rightarrow s \ell^{+} \ell^{-}$observables to confirm existing New Physics (NP) patterns (or scenarios) and to identify new ones emerging from the inclusion of the updated $\mathrm{LHCb}$ and Belle measurements of $R_{K}$ and $R_{K}$, respectively. Our analysis, updating Refs. Capdevila et al. (J Virto JHEP 1801:093, 2018) and Algueró et al. (J Matias Phys Rev D 99(7):075017, 2019) and including these new data, suggests the presence of righthanded couplings encoded in the Wilson coefficients $\mathcal{C}_{9^{\prime}} \mu$ and $\mathcal{C}_{10^{\prime} \mu}$. It also strengthens our earlier observation that a lepton flavour universality violating (LFUV) left-handed lepton coupling $\left(\mathcal{C}_{9 \mu}^{\mathrm{V}}=-\mathcal{C}_{10 \mu}^{\mathrm{V}}\right)$, often preferred from the model building point of view, accommodates the data better if lepton-flavour universal (LFU) NP is allowed, in particular in $\mathcal{C}_{9}^{\mathrm{U}}$. Furthermore, this scenario with LFU NP provides a simple and model-independent connection to the $b \rightarrow c \tau v$ anomalies, showing a preference of $\approx 7 \sigma$ with respect to the SM. It may also explain why fits to the whole set of $b \rightarrow s \ell^{+} \ell^{-}$data or to the subset of LFUV data exhibit stronger preferences for different NP scenarios. Finally, motivated by $Z^{\prime}$ models with vector-like quarks, we propose four new scenarios with LFU and LFUV NP contributions that give a very good fit to data.
\end{abstract}

\section{Introduction}

The flavour anomalies in $b \rightarrow s \ell^{+} \ell^{-}$processes are at present among the most promising signals of New Physics

\footnotetext{
a e-mail: bcapdevila@ifae.es
}

(NP). Their analyses can be efficiently and consistently performed in a model-independent effective field theory (EFT) framework (see, for instance, [1-3]), where all short-distance physics (including NP) is encoded in Wilson coefficients, i.e. the coefficients of higher-dimension operators. A central open question is then which pattern(s) in the space of the Wilson coefficients is (are) preferred by $b \rightarrow s \ell^{+} \ell^{-}$ observables. More precise measurements, in particular for the observables showing deviations from the Standard Model (SM) expectations ( $P_{5}^{\prime}$ [4], $R_{K, K^{*}, \phi}, Q_{5}$ [5]...), help us to improve the results of this EFT analysis, which can then be used as a guideline for the construction of phenomenologically accurate NP models.

In this context we present here an update and extension of our recent works in Refs. [1,2], in the light of new measurements of key observables involved in $b \rightarrow s \ell^{+} \ell^{-}$anomalies. We update the experimental value of the ratio probing lepton flavour universality (LFU) defined as $R_{K}=\frac{\mathcal{B}\left(B \rightarrow K \mu^{+} \mu^{-}\right)}{\mathcal{B}\left(B \rightarrow K e^{+} e^{-}\right)}$:

$R_{K_{\text {LHCb }}}^{[1.1,6]}=0.846_{-0.054-0.014}^{+0.060+0.016}$,
$R_{K_{\text {Belle }}}^{[1,6]}=0.98_{-0.23}^{+0.27} \pm 0.06$,
$R_{K_{\text {Belle }}^{\left[q^{2}>14.18\right]}=1.11_{-0.26}^{+0.29} \pm 0.07}$

as announced recently by the LHCb collaboration [6], corresponding to the average of Run-1 and part of Run-2 (20152016) measurements, and the Belle collaboration [7], combining the data from charged and neutral modes. The correlations with the (finely binned) measurements of $\mathcal{B}(B \rightarrow$ $K \mu^{+} \mu^{-}$) [8] are tiny and therefore neglected here. In addition the Belle collaboration has also presented new results 
for $R_{K^{*}}$, the equivalent LFU-violating (LFUV) ratio for $B \rightarrow K^{*} \ell \ell$, in three bins [9], again considering both charged and neutral channels:

$R_{K^{*}}^{[0.045,1.1]}=0.52_{-0.26}^{+0.36} \pm 0.05$,

$R_{K^{*}}^{[1.1,6]}=0.96_{-0.29}^{+0.45} \pm 0.11$,

$R_{K^{*}}^{[15,19]}=1.18_{-0.32}^{+0.52} \pm 0.10$.

Our treatment for the Belle observables within the global fit follows the same strategy as described in Ref. [1] for $Q_{4,5}$ where we introduced a nuisance parameter accounting for the relative weight of each isospin component.

We have also updated our average for $\mathcal{B}\left(B_{s} \rightarrow \mu^{+} \mu^{-}\right)$ including the latest measurement from the ATLAS collaboration [10] and taking into account the most recent lattice update of $f_{B_{s}}$ for $N_{f}=2+1+1$ simulations collected in Ref. [11].

A relatively small numerical impact of such updates has been found. As in Ref. [1], our analysis also includes the lat- est update of $P_{4,5}^{\prime}$ from the Belle collaboration [12] where the muon and electron modes are considered separately (averaging charged and neutral modes), superseding the previous measurement in Ref. [13] where an average over both leptonic modes is presented. This allows us to include an additional measurement $P_{5 \mu}^{\prime}$ (exhibiting a $2.6 \sigma$ discrepancy with respect to the SM) as well as the LFUV observable $Q_{5}$ in our analysis (see Ref. [14] for another recent analysis including this update).

In addition to updating the experimental inputs, our analysis explores new emerging directions in the parameter space spanned by the effective operators driven by data within two different frameworks. First, following Ref. [1] we assume in Sect. 2 that NP affects only muons and is thus purely LeptonFlavour Universality Violating (LFUV). In Sect. 3 we follow the complementary approach discussed in Ref. [2], where we consider the consequences of removing the frequently made hypothesis that NP is purely LFUV. We then explore the implications of allowing both LFU and LFUV NP contributions to the Wilson coefficients $\mathcal{C}_{9^{(\prime)}}$ and $\mathcal{C}_{10^{(\prime)}}$.

Table 1 Most prominent 1D patterns of NP in $b \rightarrow s \mu^{+} \mu^{-}$. Pull $\mathrm{SM}$ is quoted in units of standard deviation

\begin{tabular}{|c|c|c|c|c|c|c|c|c|}
\hline \multirow[t]{2}{*}{ 1D Hyp. } & \multicolumn{4}{|l|}{ All } & \multicolumn{4}{|l|}{ LFUV } \\
\hline & Best fit & $1 \sigma / 2 \sigma$ & Pull $_{\text {SM }}$ & p-value & Best fit & $1 \sigma / 2 \sigma$ & Pull $_{\text {SM }}$ & p-value \\
\hline $\mathcal{C}_{9 \mu}^{\mathrm{NP}}$ & -0.98 & $\begin{array}{l}{[-1.15,-0.81]} \\
{[-1.31,-0.64]}\end{array}$ & 5.6 & $65.4 \%$ & -0.89 & $\begin{array}{l}{[-1.23,-0.59]} \\
{[-1.60,-0.32]}\end{array}$ & 3.3 & $52.2 \%$ \\
\hline $\mathcal{C}_{9 \mu}^{\mathrm{NP}}=-\mathcal{C}_{10 \mu}^{\mathrm{NP}}$ & -0.46 & $\begin{array}{l}{[-0.56,-0.37]} \\
{[-0.66,-0.28]}\end{array}$ & 5.2 & $55.6 \%$ & -0.40 & $\begin{array}{l}{[-0.53,-0.29]} \\
{[-0.63,-0.18]}\end{array}$ & 4.0 & $74.0 \%$ \\
\hline $\mathcal{C}_{9 \mu}^{\mathrm{NP}}=-\mathcal{C}_{9^{\prime} \mu}$ & -0.99 & $\begin{array}{l}{[-1.15,-0.82]} \\
{[-1.31,-0.64]}\end{array}$ & 5.5 & $62.9 \%$ & -1.61 & $\begin{array}{l}{[-2.13,-0.96]} \\
{[-2.54,-0.41]}\end{array}$ & 3.0 & $42.5 \%$ \\
\hline $\mathcal{C}_{9 \mu}^{\mathrm{NP}}=-3 \mathcal{C}_{9 e}^{\mathrm{NP}}$ & -0.87 & $\begin{array}{l}{[-1.03,-0.71]} \\
{[-1.19,-0.55]}\end{array}$ & 5.5 & $61.9 \%$ & -0.66 & $\begin{array}{l}{[-0.90,-0.44]} \\
{[-1.17,-0.24]}\end{array}$ & 3.3 & $52.2 \%$ \\
\hline
\end{tabular}

The $p$-value of the SM hypothesis is $11.0 \%$ for the fit "All" and $8.0 \%$ for the fit LFUV

Table 2 Most prominent 2D patterns of NP in $b \rightarrow s \mu^{+} \mu^{-}$. The last five rows correspond to Hypothesis 1: $\left(\mathcal{C}_{9 \mu}^{\mathrm{NP}}=-\mathcal{C}_{9^{\prime} \mu}, \mathcal{C}_{10 \mu}^{\mathrm{NP}}=\mathcal{C}_{10^{\prime} \mu}\right), 2$ : $\left(\mathcal{C}_{9 \mu}^{\mathrm{NP}}=-\mathcal{C}_{9^{\prime} \mu}, \mathcal{C}_{10 \mu}^{\mathrm{NP}}=-\mathcal{C}_{10^{\prime} \mu}\right), 3:\left(\mathcal{C}_{9 \mu}^{\mathrm{NP}}=-\mathcal{C}_{10 \mu}^{\mathrm{NP}}, \mathcal{C}_{9^{\prime} \mu}=\mathcal{C}_{10^{\prime} \mu}\right), 4:\left(\mathcal{C}_{9 \mu}^{\mathrm{NP}}=-\mathcal{C}_{10 \mu}^{\mathrm{NP}}, \mathcal{C}_{9^{\prime} \mu}=-\mathcal{C}_{10^{\prime} \mu}\right)$ and 5: $\left(\mathcal{C}_{9 \mu}^{\mathrm{NP}}, \mathcal{C}_{9^{\prime} \mu}=-\mathcal{C}_{10^{\prime} \mu}\right)$

\begin{tabular}{|c|c|c|c|c|c|c|}
\hline \multirow[t]{2}{*}{ 2D Hyp. } & \multicolumn{3}{|l|}{ All } & \multicolumn{3}{|l|}{ LFUV } \\
\hline & Best fit & Pull $_{S M}$ & $\mathrm{p}$-value & Best fit & Pull $_{S M}$ & p-value \\
\hline$\left(\mathcal{C}_{9 \mu}^{\mathrm{NP}}, \mathcal{C}_{10 \mu}^{\mathrm{NP}}\right)$ & $(-0.91,0.18)$ & 5.4 & $68.7 \%$ & $(-0.16,0.56)$ & 3.4 & $76.9 \%$ \\
\hline$\left(\mathcal{C}_{9 \mu}^{\mathrm{NP}}, \mathcal{C}_{7^{\prime}}\right)$ & $(-1.00,0.02)$ & 5.4 & $67.9 \%$ & $(-0.90,-0.04)$ & 2.9 & $55.1 \%$ \\
\hline$\left(\mathcal{C}_{9 \mu}^{\mathrm{NP}}, \mathcal{C}_{9^{\prime} \mu}\right)$ & $(-1.10,0.55)$ & 5.7 & $75.1 \%$ & $(-1.79,1.14)$ & 3.4 & $76.1 \%$ \\
\hline$\left(\mathcal{C}_{9 \mu}^{\mathrm{NP}}, \mathcal{C}_{10^{\prime} \mu}\right)$ & $(-1.14,-0.35)$ & 5.9 & $78.6 \%$ & $(-1.88,-0.62)$ & 3.8 & $91.3 \%$ \\
\hline$\left(\mathcal{C}_{9 \mu}^{\mathrm{NP}}, \mathcal{C}_{9 e}^{\mathrm{NP}}\right)$ & $(-1.05,-0.23)$ & 5.3 & $66.2 \%$ & $(-0.73,0.16)$ & 2.8 & $52.3 \%$ \\
\hline Hyp. 1 & $(-1.06,0.26)$ & 5.7 & $75.7 \%$ & $(-1.62,0.29)$ & 3.4 & $77.6 \%$ \\
\hline Hyp. 2 & $(-0.97,0.09)$ & 5.3 & $65.2 \%$ & $(-1.95,0.25)$ & 3.2 & $66.6 \%$ \\
\hline Hyp. 3 & $(-0.47,0.06)$ & 4.8 & $55.7 \%$ & $(-0.39,-0.13)$ & 3.4 & $76.2 \%$ \\
\hline Hyp. 4 & $(-0.49,0.12)$ & 5.0 & $59.3 \%$ & $(-0.48,0.17)$ & 3.6 & $84.3 \%$ \\
\hline Hyp. 5 & $(-1.14,0.24)$ & 5.9 & $78.7 \%$ & $(-2.07,0.52)$ & 3.9 & $92.5 \%$ \\
\hline
\end{tabular}


Motivated by the new emerging directions in the LFUV case we also extend our analysis of NP scenarios to allow for the presence of LFU NP right handed-currents (RHC). In Sect. 4 , we focus on a particular scenario (scenario 8) which can, within an EFT framework, link the flavour anomalies in $b \rightarrow s \ell^{+} \ell^{-}$and $b \rightarrow c \ell v$ processes. Furthermore, we consider new patterns, motivated by $Z^{\prime}$ models with vector-like quarks, which naturally predict LFU effects in $\mathcal{C}_{10^{(\prime)}}$ complemented by LFUV ones. Finally, we sum up our results in Sect. 5. An appendix is devoted to the description of the correlations obtained for the various Wilson coefficients in the most relevant scenarios considered in this article.

\section{Global fits in presence of LFUV NP}

We start by considering the fits for NP scenarios which affect muon modes only. Tables 1, 2 and 3 and Fig. 1 update the corresponding tables and figures of Ref. [1] based on fits to the full set of data ("All") or restricted to quantities assessing LFUV. While we do not observe any significant difference in the 1D scenarios with "All" data compared to Ref. [1], some of the Pulls (with respect to the SM) for the LFUV 1D fits get reduced by half a standard deviation. A few other comments are in order:

Table 31 and $2 \sigma$ confidence intervals for the NP contributions to Wilson coefficients in the 6D hypothesis allowing for NP in $b \rightarrow s \mu^{+} \mu^{-}$ operators dominant in the SM and their chirally-flipped counterparts, for the fit "All"

\begin{tabular}{lllllll}
\hline & $\mathcal{C}_{7}^{\mathrm{NP}}$ & $\mathcal{C}_{9 \mu}^{\mathrm{NP}}$ & $\mathcal{C}_{10 \mu}^{\mathrm{NP}}$ & $\mathcal{C}_{7^{\prime}}$ & $\mathcal{C}_{9^{\prime} \mu}$ & $\mathcal{C}_{10^{\prime} \mu}$ \\
\hline Best fit & +0.01 & -1.10 & +0.15 & +0.02 & +0.36 & -0.16 \\
$1 \sigma$ & {$[-0.01,+0.05]$} & {$[-1.28,-0.90]$} & {$[-0.00,+0.36]$} & {$[-0.00,+0.05]$} & {$[-0.14,+0.87]$} & {$[-0.39,+0.13]$} \\
$2 \sigma$ & {$[-0.03,+0.06]$} & {$[-1.44,-0.68]$} & {$[-0.12,+0.56]$} & {$[-0.02,+0.06]$} & {$[-0.49,+1.23]$} & {$[-0.58,+0.33]$} \\
\hline
\end{tabular}

The Pull ${ }_{\mathrm{SM}}$ is $5.1 \sigma$ and the $p$-value is $81.6 \%$
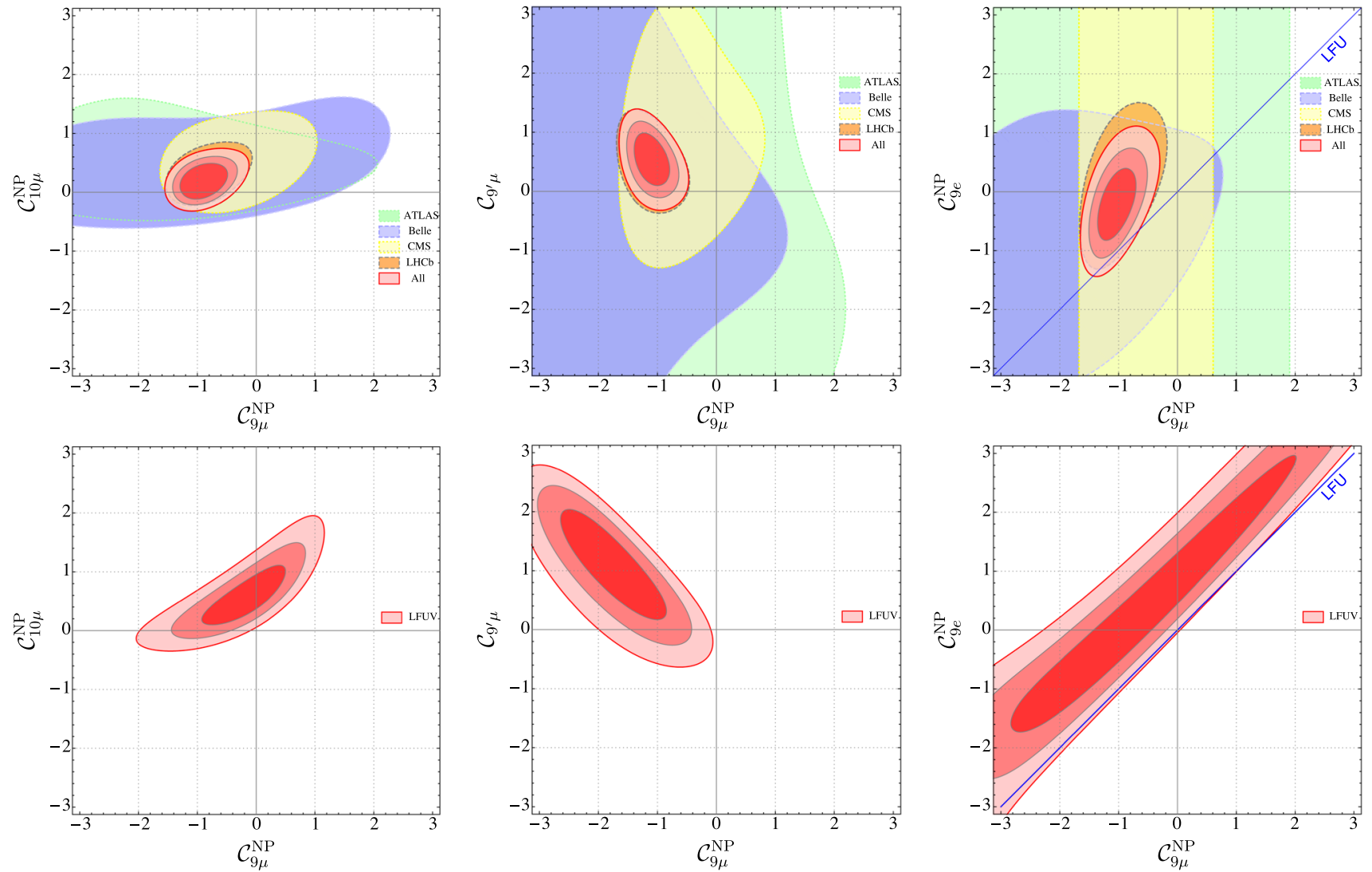

Fig. 1 From left to right: allowed regions in the $\left(\mathcal{C}_{9 \mu}^{\mathrm{NP}}, \mathcal{C}_{10 \mu}^{\mathrm{NP}}\right),\left(\mathcal{C}_{9 \mu}^{\mathrm{NP}}, \mathcal{C}_{9^{\prime} \mu}\right)$ and $\left(\mathcal{C}_{9 \mu}^{\mathrm{NP}}, \mathcal{C}_{9 e}^{\mathrm{NP}}\right)$ planes for the corresponding $2 \mathrm{D}$ hypotheses, using all available data (fit "All”) upper row or LFUV fit lower row 
Table 4 Coefficients for the polynomial parameterisation of the numerator and denominator of $R_{K}^{[1.1,6]}$ in the vicinity of the SM point

\begin{tabular}{ccccccccccc}
\hline$\alpha_{0 \mu}$ & $\alpha_{1 \mu}$ & $\alpha_{2 \mu}$ & $\alpha_{3 \mu}$ & $\alpha_{4 \mu}$ & $\alpha_{5 \mu}$ & $\alpha_{6 \mu}$ & $\alpha_{7 \mu}$ & $\alpha_{8 \mu}$ & $\alpha_{9 \mu}$ & $\alpha_{10 \mu}$ \\
\hline 4.00 & 0.92 & 0.12 & 0.92 & 0.12 & 0.24 & -1.06 & 0.12 & -1.06 & 0.12 & 0.25 \\
\hline$\alpha_{0 e}$ & $\alpha_{1 e}$ & $\alpha_{2 e}$ & $\alpha_{3 e}$ & $\alpha_{4 e}$ & $\alpha_{5 e}$ & $\alpha_{6 e}$ & $\alpha_{7 e}$ & $\alpha_{8 e}$ & $\alpha_{9 e}$ & $\alpha_{10 e}$ \\
\hline 3.99 & 0.92 & 0.12 & 0.92 & 0.12 & 0.24 & -1.05 & 0.12 & -1.05 & 0.12 & 0.24 \\
\hline
\end{tabular}

1. The scenario $\mathcal{C}_{9 \mu}^{\mathrm{NP}}=-\mathcal{C}_{9^{\prime} \mu}$, which favours a SM-like value of $R_{K}^{[1.1,6]}[2,15]$, has an increased significance in the "All" fit compared to our earlier analysis.

2. The scenario $\mathcal{C}_{9 \mu}^{\mathrm{NP}}$ has the largest $p$-value in the "All" fit while $\mathcal{C}_{9 \mu}^{\mathrm{NP}}=-\mathcal{C}_{10 \mu}^{\mathrm{NP}}$ has the largest $p$-value in the LFUV fit, a difference which can be solved through the introduction of LFU NP (see Ref. [2] and next section).

3. The best-fit point for the scenario $\mathcal{C}_{9 \mu}^{\mathrm{NP}}$ coincides now in the "All" and LFUV fits.

4. The scenario with only $\mathcal{C}_{10 \mu}^{\mathrm{NP}}$ has a significance in the "All" fit of only $4.0 \sigma$ level and $3.9 \sigma$ for the LFUV fit, which explains its absence from Table 1 as happens in Ref. [1].

Concerning the 2D scenarios collected in Table 2, the same picture arises as in Ref. [1], except that $\mathcal{C}_{9 e}^{\mathrm{NP}}$ is now basically zero and small contributions to RHC seem slightly favoured $\left(\mathcal{C}_{9^{\prime} \mu}>0, \mathcal{C}_{10^{\prime} \mu}<0\right) .{ }^{1}$ Indeed, these RHC contributions tend to increase the value of $R_{K}^{[1.1,6]}$ while $\mathcal{C}_{9 \mu}^{\mathrm{NP}}<0$ tend to decrease it as can be seen from the explicit expression of $R_{K}^{[1.1,6]}=A_{\mu} / A_{e}$ where the numerator and the denominator can be given by an approximate polynomial parameterisation near the SM point

$$
\begin{aligned}
A_{\ell}= & \alpha_{0 \ell}+\alpha_{1 \ell} \mathcal{C}_{9 \ell}^{\mathrm{NP}}+\alpha_{2 \ell}\left(\mathcal{C}_{9 \ell}^{\mathrm{NP}}\right)^{2}+\alpha_{3 \ell} \mathcal{C}_{9^{\prime} \ell} \\
& +\alpha_{4 \ell}\left(\mathcal{C}_{9^{\prime} \ell}\right)^{2}+\alpha_{5 \ell} \mathcal{C}_{9 \ell}^{\mathrm{NP}} \mathcal{C}_{9^{\prime} \ell} \\
& +\alpha_{6 \ell} \mathcal{C}_{10 \ell}^{\mathrm{NP}}+\alpha_{7 \ell}\left(\mathcal{C}_{10 \ell}^{\mathrm{NP}}\right)^{2} \\
& +\alpha_{8 \ell} \mathcal{C}_{10^{\prime} \ell}+\alpha_{9 \ell}\left(\mathcal{C}_{10^{\prime} \ell}\right)^{2}+\alpha_{10 \ell} \mathcal{C}_{10 \ell}^{\mathrm{NP}} \mathcal{C}_{10^{\prime} \ell}
\end{aligned}
$$

with the coefficients provided in Table 4 (for linearised expressions, see Refs. [2,16]). We introduce a new Hyp. 5 in Table 2. The comparison between Hyps. 4 and 5 shows that the scenario $\mathcal{C}_{9^{\prime} \mu}=-\mathcal{C}_{10^{\prime} \mu}$ (left-handed lepton coupling for right-handed quarks) prefers to be associated with $\mathcal{C}_{9 \mu}^{\mathrm{NP}}$ (vector lepton coupling for left-handed quarks) rather than $\mathcal{C}_{9 \mu}^{\mathrm{NP}}=-\mathcal{C}_{10 \mu}^{\mathrm{NP}}$ (left-handed lepton coupling for left-handed quarks). Finally, no significant changes are observed in the $6 \mathrm{D}$ fit, except for the slight increase in the Pull $\mathrm{SM}$, see Table 3.

\footnotetext{
${ }^{1}$ Interestingly, these small contributions also reduce slightly the mild tension between $P_{5}^{\prime}$ at large and low recoils pointed out in Ref. [15] compared to the scenario with only $\mathcal{C}_{9 \mu}^{\mathrm{NP}}$.
}

With the updated data, little change is observed among the preferred 2D NP models. Nevertheless, with an $R_{K}^{[1.1,6]}$ value closer to one, scenarios with right-handed currents (RHC), namely $\left(\mathcal{C}_{9 \mu}^{\mathrm{NP}}, \mathcal{C}_{9^{\prime} \mu}\right)$ and $\left(\mathcal{C}_{9 \mu}^{\mathrm{NP}}, \mathcal{C}_{10^{\prime} \mu}\right)$, seem to emerge. The first scenario is naturally generated in a $Z^{\prime}$ model with opposite couplings to right-handed and left-handed quarks and was proposed in Ref. [17] within the context of a gauged $L_{\mu}-L_{\tau}$ symmetry with vector-like quarks. The latter (of masses $m_{D}$ and $m_{Q}$ ) are charged under $L_{\mu}-L_{\tau}$ and have the same SM quantum numbers as right-handed down quarks and left-handed quark doublets, respectively. The vector-like quarks couple to the SM ones and to a scalar $\phi$ which breaks the $L_{\mu}-L_{\tau}$ symmetry with couplings $Y^{D, Q}$. We show the update of Fig. 2 of Ref. [17] assuming $Y^{D, Q}=1$ in Fig. 2. Since the current fit allows for $\mathcal{C}_{9^{\prime} \mu}=0$ at the two sigma level, the $S U(2)$ singlet vector-like quark can still be decoupled [18].

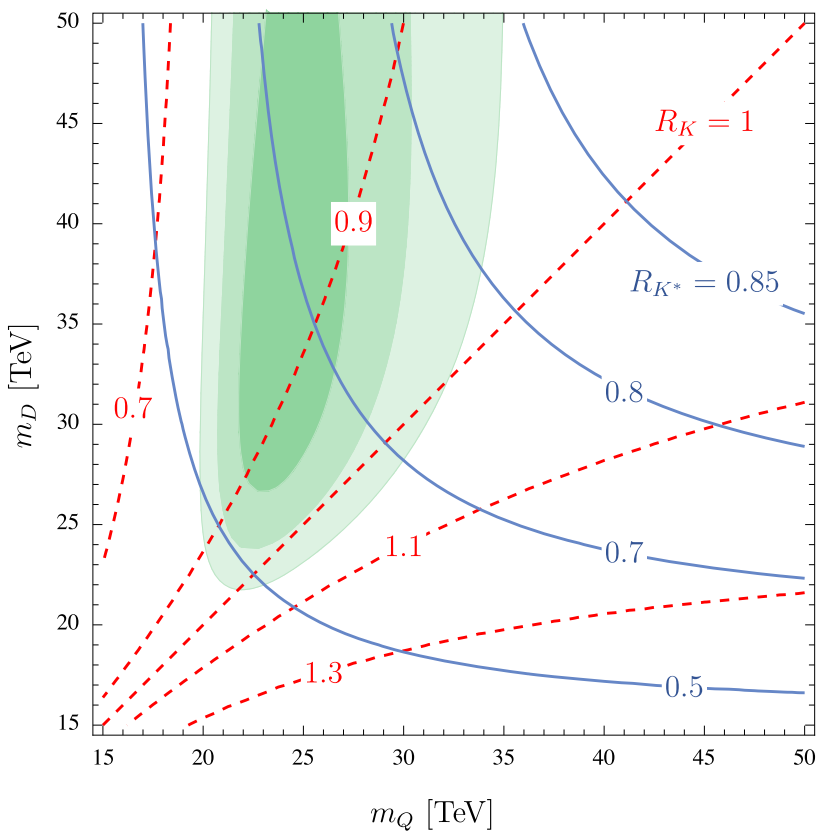

Fig. 2 Preferred regions (at the 1,2 and $3 \sigma$ level) for the $L_{\mu}-L_{\tau}$ model of Ref. [17] from $b \rightarrow s \ell^{+} \ell^{-}$data (green) in the $\left(m_{Q}, m_{D}\right)$ plane with $Y^{D, Q}=1$. The contour lines denote the predicted values for $R_{K}^{[1.1,6]}$ (red, dashed) and $R_{K^{*}}^{[1.1,6]}$ (blue, solid) 


\section{Global fits in presence of LFUV and LFU NP}

We turn to scenarios that allow also for the presence of LFU NP $[2,15]$ (in addition to LFUV contributions to muons only), leading to the value of the Wilson coefficients

$\mathcal{C}_{i e}=\mathcal{C}_{i}^{\mathrm{U}}, \quad \mathcal{C}_{i \mu}=\mathcal{C}_{i}^{\mathrm{U}}+\mathcal{C}_{i}^{\mathrm{V}}$.

(with $i=9,10$ ) for $b \rightarrow s e^{+} e^{-}$and $b \rightarrow s \mu^{+} \mu^{-}$transitions respectively.

We update some of the scenarios considered in Ref. [2] in Table 5. Concerning new directions in parameter space we allow for RHC, motivated by the results of the previous section, and focus on scenarios that could be fairly easily obtained in simple NP models.

With the updated experimental inputs, we confirm our earlier result [2] that a LFUV left-handed lepton coupling structure (corresponding to $\mathcal{C}_{9}^{\mathrm{V}}=-\mathcal{C}_{10}^{\mathrm{V}}$ and preferred from a model-building point of view) yields a better description of data with the addition of LFU-NP in the coefficients $\mathcal{C}_{9,10}$, as shown by the scenarios 6,8 in Table 5 with $p$-values larger than $70 \%$.

We observe a very slight decrease in significance for the scenarios 5-7, with the exception of scenario 8 which exhibits one of the most significant pulls with respect to the SM.

Scenario 8 of Ref. [2] can actually be realized via off-shell photon penguins [19] in a leptoquark model explaining also $b \rightarrow c \tau v$ data (we will return to this point in the following section).

Updated plots of the 2D LFU-LFUV scenarios discussed in Ref. [2] are shown in Fig. 3.

The new scenarios $9-13$ are characterized by a $\mathcal{C}_{10\left(^{\prime}\right)}^{\mathrm{U}}$ contribution. This arises naturally in models with modified $Z$ couplings (to a good approximation $\mathcal{C}_{9\left(^{\prime}\right)}^{\mathrm{U}}$ can be neglected). The pattern of scenario 9 occurs in Two-Higgs-Doublet models where this flavour universal effect can be supplemented by a $\mathcal{C}_{9}^{\mathrm{V}}=-\mathcal{C}_{10}^{\mathrm{V}}$ effect [20].

In case of scenarios 11-13, one can invoke models with vector-like quarks where modified $Z$ couplings are even

Table 5 Most prominent patterns for LFU and LFUV NP contributions from Fit "All"

\begin{tabular}{|c|c|c|c|c|c|c|}
\hline \multicolumn{2}{|l|}{ Scenario } & \multirow{2}{*}{$\frac{\text { Best-fit point }}{-0.36}$} & \multirow{2}{*}{$\frac{1 \sigma}{[-0.86,+0.10]}$} & \multirow{2}{*}{$\frac{2 \sigma}{[-1.41,+0.52]}$} & \multirow{2}{*}{$\frac{\text { Pull }_{\mathrm{SM}}}{5.2}$} & \multirow{2}{*}{$\frac{\mathrm{p} \text {-value }}{71.2 \%}$} \\
\hline Scenario 5 & $\mathcal{C}_{9 \mu}^{\mathrm{V}}$ & & & & & \\
\hline & $\mathcal{C}_{10 \mu}^{\mathrm{V}}$ & +0.67 & {$[+0.24,+1.03]$} & {$[-1.73,+1.36]$} & & \\
\hline & $\mathcal{C}_{9}^{\mathrm{U}}=\mathcal{C}_{10}^{\mathrm{U}}$ & -0.59 & {$[-0.90,-0.12]$} & {$[-1.13,+0.68]$} & & \\
\hline \multirow[t]{2}{*}{ Scenario 6} & $\mathcal{C}_{9 \mu}^{\mathrm{V}}=-\mathcal{C}_{10 \mu}^{\mathrm{V}}$ & -0.50 & {$[-0.61,-0.38]$} & {$[-0.72,-0.28]$} & 5.5 & $71.0 \%$ \\
\hline & $\mathcal{C}_{9}^{\mathrm{U}}=\mathcal{C}_{10}^{\mathrm{U}}$ & -0.38 & {$[-0.52,-0.22]$} & {$[-0.64,-0.06]$} & & \\
\hline \multirow[t]{2}{*}{ Scenario 7} & $\mathcal{C}_{9 \mu}^{\mathrm{V}}$ & -0.78 & {$[-1.11,-0.47]$} & {$[-1.45,-0.18]$} & 5.3 & $66.2 \%$ \\
\hline & $\mathcal{C}_{9}^{\mathrm{U}}$ & -0.20 & {$[-0.57,+0.18]$} & {$[-0.92,+0.55]$} & & \\
\hline \multirow[t]{2}{*}{ Scenario 8} & $\mathcal{C}_{9 \mu}^{\mathrm{V}}=-\mathcal{C}_{10 \mu}^{\mathrm{V}}$ & -0.30 & {$[-0.42,-0.20]$} & {$[-0.53,-0.10]$} & 5.7 & $75.2 \%$ \\
\hline & $\mathcal{C}_{9}^{\mathrm{U}}$ & -0.74 & {$[-0.96,-0.51]$} & {$[-1.15,-0.25]$} & & \\
\hline \multirow[t]{2}{*}{ Scenario 9} & $\mathcal{C}_{9 \mu}^{\mathrm{V}}=-\mathcal{C}_{10 \mu}^{\mathrm{V}}$ & -0.57 & {$[-0.73,-0.41]$} & {$[-0.87,-0.28]$} & 5.0 & $60.2 \%$ \\
\hline & $\mathcal{C}_{10}^{\mathrm{U}}$ & -0.34 & {$[-0.60,-0.07]$} & {$[-0.84,+0.18]$} & & \\
\hline \multirow[t]{2}{*}{ Scenario 10} & $\mathcal{C}_{9 \mu}^{\mathrm{V}}$ & -0.95 & {$[-1.13,-0.76]$} & {$[-1.30,-0.57]$} & 5.5 & $69.5 \%$ \\
\hline & $\mathcal{C}_{10}^{\mathrm{U}}$ & +0.27 & {$[0.08,0.47]$} & {$[-0.09,0.66]$} & & \\
\hline \multirow[t]{2}{*}{ Scenario 11} & $\mathcal{C}_{9 \mu}^{\mathrm{V}}$ & -1.03 & {$[-1.22,-0.84]$} & {$[-1.38,-0.65]$} & 5.6 & $73.6 \%$ \\
\hline & $\mathcal{C}_{10^{\prime}}^{\mathrm{U}}$ & -0.29 & {$[-0.47,-0.12]$} & {$[-0.63,0.05]$} & & \\
\hline \multirow[t]{2}{*}{ Scenario 12} & $\mathcal{C}_{9^{\prime} \mu}^{\mathrm{V}}$ & -0.03 & {$[-0.22,0.15]$} & {$[-0.40,0.32]$} & 1.6 & $15.7 \%$ \\
\hline & $\mathcal{C}_{10}^{\mathrm{U}}$ & +0.41 & {$[0.21,0.63]$} & {$[0.02,0.83]$} & & \\
\hline \multirow[t]{4}{*}{ Scenario 13} & $\mathcal{C}_{9 \mu}^{\mathrm{V}}$ & -1.11 & {$[-1.28,-0.91]$} & {$[-1.41,-0.71]$} & 5.4 & $78.7 \%$ \\
\hline & $\mathcal{C}_{9^{\prime} \mu}^{\mathrm{V}}$ & +0.53 & {$[0.24,0.83]$} & {$[-0.10,1.11]$} & & \\
\hline & $\mathcal{C}_{10}^{\mathrm{U}}$ & +0.24 & {$[0.01,0.48]$} & {$[-0.21,0.69]$} & & \\
\hline & $\mathcal{C}_{10^{\prime}}^{\mathrm{U}}$ & -0.04 & {$[-0.28,0.20]$} & {$[-0.48,0.42]$} & & \\
\hline
\end{tabular}

Scenarios 5-8 were introduced in Ref. [2]. Scenarios 9 (motivated by 2 HDMs [20]) and 10-13 (motivated by $Z^{\prime}$ models with vector-like quarks [21]) are new 
Fig. 3 Updated plots of Ref. [2] corresponding to scenarios $6,7,8$ and the new scenario 9
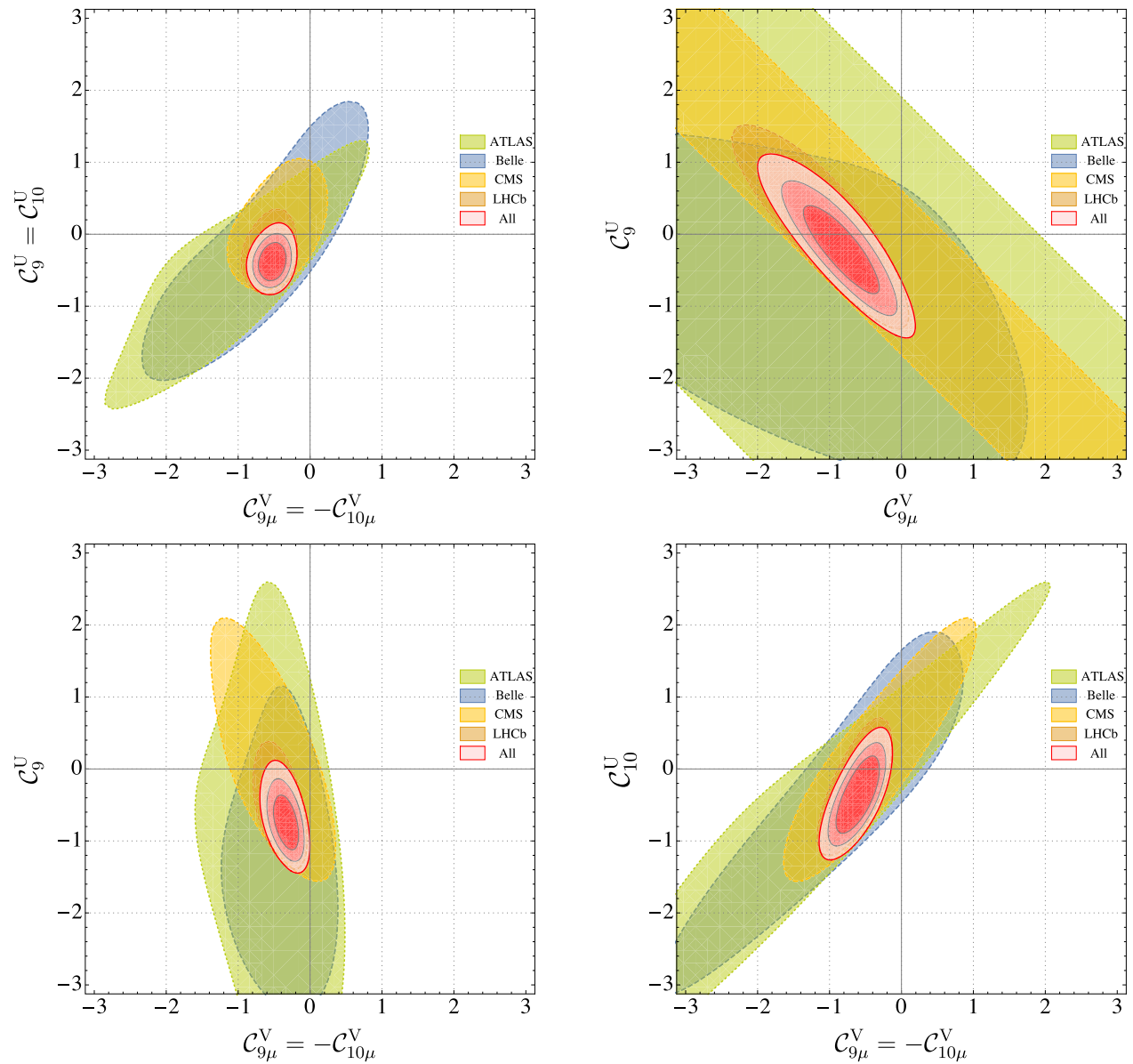

induced at tree level. The LFU effect in $\mathcal{C}_{10\left({ }^{\prime}\right)}^{\mathrm{U}}$ can be accompanied by a $\mathcal{C}_{9,10\left(^{\prime}\right)}^{\mathrm{V}}$ effect from $Z^{\prime}$ exchange [21]. Vectorlike quarks with the quantum numbers of right-handed down quarks (left-handed quarks doublets) generate effect in $\mathcal{C}_{10}^{\mathrm{U}}$ and $\mathcal{C}_{9^{\prime}}^{\mathrm{V}}\left(\mathcal{C}_{10\left(^{\prime}\right)}^{\mathrm{U}}\right.$ and $\left.\mathcal{C}_{9}^{\mathrm{V}}\right)$ for a $Z^{\prime}$ boson with vector couplings to muons [21].

The comparison of scenarios 10 and 12 illustrates that $\mathcal{C}_{9 \mu}^{\mathrm{V}}$ plays an important role in LFU NP scenarios and can- not be swapped for its chirally-flipped counterpart without consequences. Finally, the allowed regions for the new LFU scenarios are displayed in Fig. 4.

\section{Model-independent connection to $b \rightarrow c \ell v$}

In complement with the above EFT analysis, we focus now on the NP interpretation of scenario 8. Indeed, this sce-
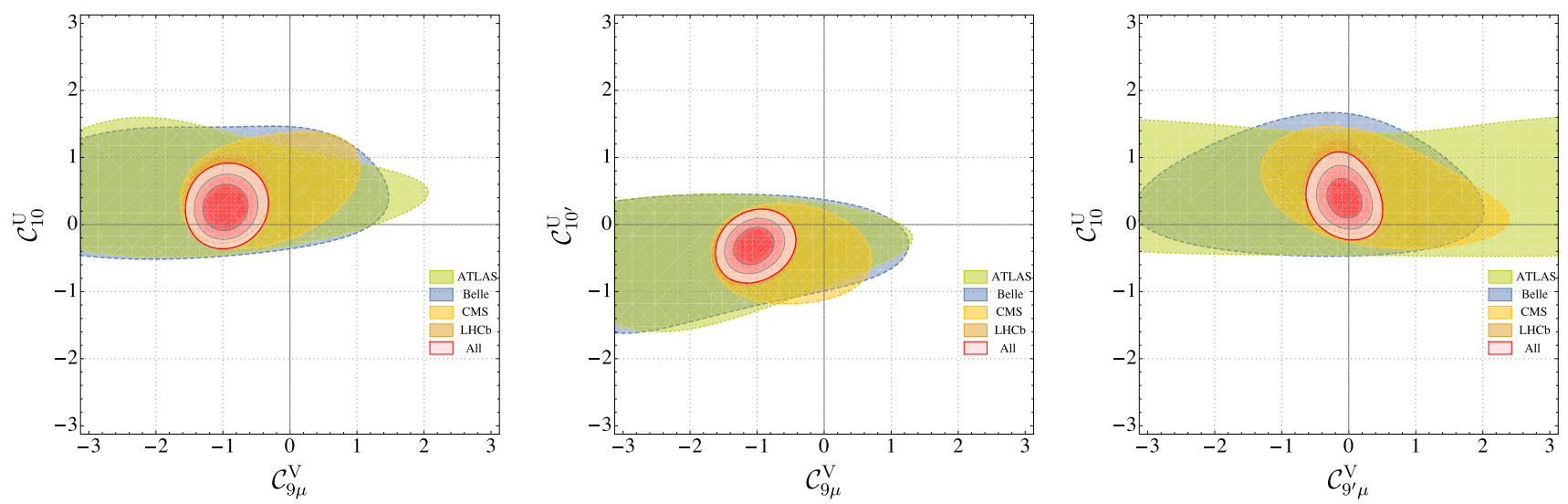

Fig. 4 Updated plots of Ref. [2] corresponding to the new scenarios 10, 11, 12 


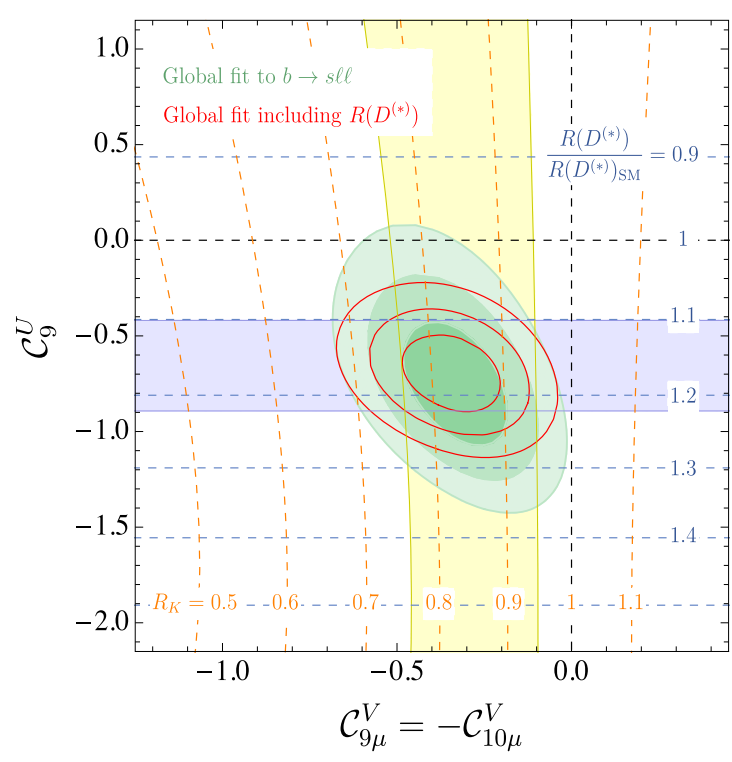

Fig. 5 Left: preferred regions at the 1,2 and $3 \sigma$ level (green) in the $\left(\mathcal{C}_{9 \mu}^{\mathrm{V}}=-\mathcal{C}_{10 \mu}^{\mathrm{V}}, \mathcal{C}_{9}^{\mathrm{U}}\right)$ plane from $b \rightarrow s \ell^{+} \ell^{-}$data. The red contour lines show the corresponding regions once $R_{D^{(*)}}$ is included in the fit (for $\Lambda=2 \mathrm{TeV}$ ). The horizontal blue (vertical yellow) band is consistent with $R_{D^{(*)}}\left(R_{K}\right)$ at the $2 \sigma$ level and the contour lines show the

nario allows for a model-independent connection between the anomalies in $b \rightarrow s \ell^{+} \ell^{-}$and those in $b \rightarrow c \tau v$, which are now at the $3.1 \sigma$ level [22].

Such a correlation arises in the SMEFT scenario where $\mathcal{C}^{(1)}=\mathcal{C}^{(3)}$ expressed in terms of gauge-invariant dimension6 operators $[23,24]$. This scenario stems naturally from models with an $S U$ (2) singlet vector leptoquark [25-27]. The operator involving-third generation leptons explains $R_{D^{(*)}}$ and the one involving the second generation gives a LFUV effect in $b \rightarrow s \mu^{+} \mu^{-}$processes. The constraint from $b \rightarrow$ $c \tau v$ and $S U(2)_{L}$ invariance leads generally to large contributions to the operator $\bar{s} \gamma^{\mu} P_{L} b \bar{\tau} \gamma_{\mu} P_{L} \tau$, which enhances $b \rightarrow s \tau^{+} \tau^{-}$processes [24], but also mixes into $\mathcal{O}_{9}$ and generates $\mathcal{C}_{9}^{\mathrm{U}}$ at $\mu=m_{b}$ [19]. Note that not all models addressing the charged and neutral current anomalies simultaneously have an anarchic flavour structure. In fact, in the case of alignment in the down-sector $[29,30]$ one does not find large effects in $b \rightarrow s \tau^{+} \tau^{-}$or $\mathcal{C}_{9}^{\mathrm{U}}$.

Therefore, scenario 8 is reproduced in this setup with an additional correlation between $\mathcal{C}_{9}^{\mathrm{U}}$ and $R_{D^{(*)}}$. Assuming a generic flavour structure so that small CKM elements can be neglected $[19,24]$, we get

$\mathcal{C}_{9}^{\mathrm{U}} \approx 7.5\left(1-\sqrt{\frac{R_{D^{(*)}}}{R_{D^{(*)} \mathrm{SM}}}}\right)\left(1+\frac{\log \left(\Lambda^{2} /\left(1 \mathrm{TeV}^{2}\right)\right)}{10.5}\right)$.

Realizations of this scenario in specific NP models yield also an effect in $\mathcal{C}_{7}$ generally [19]. However, since this effect is model dependent (and in fact small in some UV complete

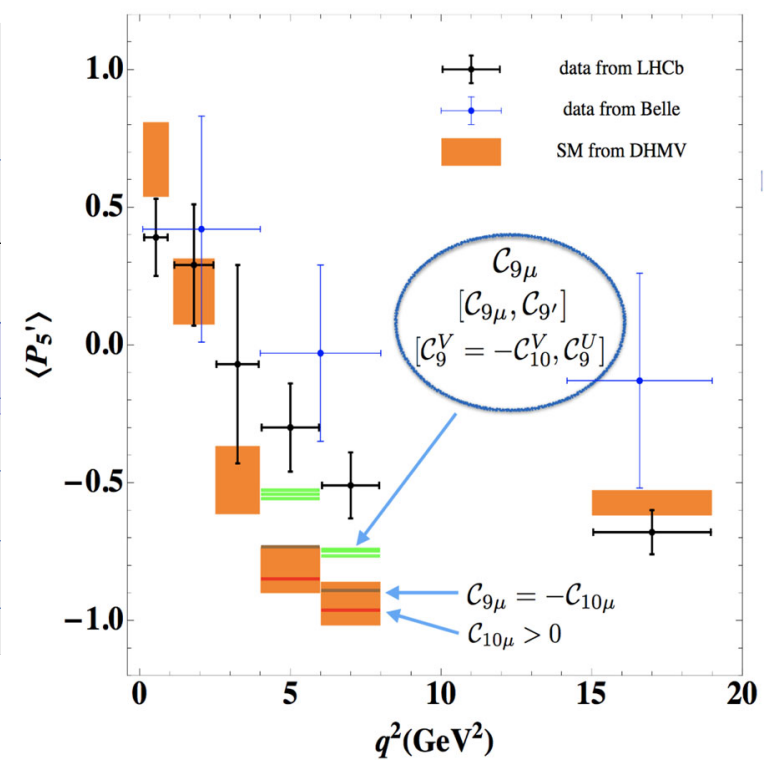

predicted values for these ratios. Right: Impact of favoured NP scenarios on the observable $P_{5}^{\prime}$. Only central values for the NP scenarios are displayed. The most interesting scenarios cluster together while traditional scenarios like $\mathcal{C}_{9 \mu}=-\mathcal{C}_{10 \mu}$ or the scenario $\mathcal{C}_{10 \mu}$ considered in Ref. [28] fail to explain this anomaly

models [31,32]), we neglect it here, leading to the left plot in Fig. 5, where we include the recent update of Ref. [33] to draw the band for $R_{D(*)}$. Note that this scenario has a pull of $7.0 \sigma$ due to the inclusion of $R_{D^{(*)}}$, which increases $\Delta \chi^{2}$ by $\sim 20$.

\section{Conclusions}

In summary, including recent updates $\left(R_{K}, R_{K^{*}}\right.$ and $\mathcal{B}\left(B_{S} \rightarrow\right.$ $\left.\mu^{+} \mu^{-}\right)$) our global model-independent analysis yields a very similar picture to the one previously found in Refs. [1,2] for the various NP scenarios of interest with some important peculiarities. In presence of LFUV NP contributions only, the 1D fits to "All" observables remain basically unchanged showing the preference for $\mathcal{C}_{9 \mu}^{\mathrm{NP}}$ scenario over $\mathcal{C}_{9 \mu}^{\mathrm{NP}}=-\mathcal{C}_{10 \mu}^{\mathrm{NP}}$. If only LFUV observables are considered the situation is reversed, as already found in Ref. [1], but now with an increased gap between the significances. This difference between the preferred hypotheses, depending on the data set used, can be solved introducing LFU NP contributions [2].

The main differences arise for the 2D scenarios: the cases including RHC, $\left(\mathcal{C}_{9 \mu}^{\mathrm{NP}}, \mathcal{C}_{10^{\prime} \mu}\right),\left(\mathcal{C}_{9 \mu}^{\mathrm{NP}}, \mathcal{C}_{9^{\prime} \mu}\right)$ or $\left(\mathcal{C}_{9 \mu}^{\mathrm{NP}}, \mathcal{C}_{9^{\prime} \mu}=\right.$ $\left.-\mathcal{C}_{10^{\prime} \mu}\right)$, can accommodate better the recent updates, which enhances the significance of these scenarios compared to Ref. [1], pointing to new patterns including RHC. A more precise experimental measurement of the observable $P_{1}[34,35]$ would be very useful to confirm or not the presence of RHC NP encoded in $\mathcal{C}_{9^{\prime} \mu}$ and $\mathcal{C}_{10^{\prime} \mu}$. 
We also observe interesting changes in the $2 \mathrm{D}$ fits in the presence of LFU NP, where new scenarios (not considered in Ref. [2]) give a good fit to data with $\mathcal{C}_{10^{(\prime)}}^{\mathrm{U}}$ and additional LFUV contributions. For example scenario 11 $\left(\mathcal{C}_{9 \mu}^{\mathrm{V}}, \mathcal{C}_{10^{\prime} \mu}\right)$ can accommodate $b \rightarrow s \ell^{+} \ell^{-}$data very well, at the same level as scenario 8. Scenarios including LFU NP in left-handed currents (discussed in Ref. [2]) stay practically unchanged but with some preference for scenarios 6 and 8 , which have a $(V-A)$ structure for the LFUV-NP and a $V$ or $(V+A)$ structure for the LFU-NP. Furthermore, we have included additional scenarios 9 and 10 that exhibit a significance of $5.0 \sigma$ and $5.5 \sigma$ respectively.

We note that the amount of LFU NP is sensitive to the structure of the LFUV component. For instance, in scenario $7\left(\mathcal{C}_{9 \mu}^{\mathrm{V}}\right.$ and $\left.\mathcal{C}_{9}^{\mathrm{U}}\right)$ the LFU component is negligible at its best fit point. On the contrary, if the LFUV-NP has a $(V-A)$ structure, the LFU-NP component $\left(\mathcal{C}_{9}^{\mathrm{U}}\right)$ is large, as illustrated by scenarios 6, 8 and 9. Scenarios with NP in RHC (either LFU or LFUV) prefer such contributions at the $2 \sigma$ level (see scenarios 11 and 13) with the exception of scenario 12 with negligible $\mathcal{C}_{9^{\prime} \mu}^{\mathrm{V}}$. The new values of $R_{K}$ and $R_{K^{*}}$ seem thus to open a window for RHC contributions while the new $\mathcal{B}\left(B_{s} \rightarrow \mu \mu\right)$ update (theory and experiment) helps only marginally scenarios with $\mathcal{C}_{10 \mu}^{\mathrm{NP}}$.

Finally, we showed that scenario 8, which allows for a model-independent connection between the $b \rightarrow c \tau v$ anomalies and the ones in $b \rightarrow s \ell^{+} \ell^{-}$, can explain all data consistently and is preferred over the SM by $7 \sigma$.

Figure 5 illustrates the impact on the largest anomaly $\left(P_{5}^{\prime}\right)$ of some of the most significant scenarios. Interestingly, several of the scenarios currently favoured cluster around the same values for the bins showing deviations with respect to the SM.

We have thus identified a number of NP scenarios with similarly good $p$-values and pulls with respect to the SM, which are able to reproduce the $b \rightarrow s \ell^{+} \ell^{-}$data very well. Hierarchies among these scenarios can be identified, but additional data and reduced uncertainties are required to come to a final conclusion. The full exploitation of LHC run-2 data by the LHCb experiment (as well as by ATLAS and CMS) and the forthcoming results from the Belle and Belle II collaborations are expected to improve the situation very significantly in the forthcoming years, helping us to pin down the actual NP pattern hinted at by the $b \rightarrow s \ell^{+} \ell^{-}$anomalies currently observed and to build accurate phenomenological models to be confirmed through other experimental probes such as direct production experiments.

Note added After the completion of this work, several global analyses have been performed to assess NP scenarios affecting $b \rightarrow s \ell^{+} \ell^{-}$processes [14,28,36,37]. They agree well with our findings, with small differences stemming mainly from slightly different theoretical approaches as well as theoretical and experimental inputs. The improve- ment brought by RHC has been observed in Refs. [14,36], whereas the interest of LFU NP contributions is also identified in Refs. $[14,28,38]$. Most of the analyses observe that the slight deviation from $\mathcal{B}\left(B_{s} \rightarrow \mu^{+} \mu^{-}\right)$plays no specific role in the global fit [36,37], apart from Ref. [28]. In the latter analysis, the significance of a scenario with only $\mathcal{C}_{10 \mu}^{\mathrm{NP}}$ is much more important than in our case, and the hierarchies between the significances of 2D scenarios is different. After discussion with the authors of Ref. [28], this difference comes from their inclusion of $B_{S}-\bar{B}_{S}$ mixing and the assumption that $\Delta F=2$ observables are purely governed by the SM, which helps them sharpening the prediction for $\mathcal{B}\left(B_{s} \rightarrow \mu^{+} \mu^{-}\right)$and increase the weight of this observable in the fit. Our present analysis does not rely on this strong hypothesis, which should be contrasted with the fact that most models invoked to explain $b \rightarrow s \ell^{+} \ell^{-}$anomalies typically affect also $\Delta F=2$ observables.

Acknowledgements This work received financial support from European Regional Development Funds under the Spanish Ministry of Science, Innovation and Universities (Projects FPA2014-55613-P and FPA2017-86989-P) and from the Agency for Management of University and Research Grants of the Government of Catalonia (project SGR 1069) [MA, BC, PM, JM] and from European Commission (Grant Agreements 690575, 674896 and 69219) [SDG]. The work of PM is supported by the Beatriu de Pinos postdoctoral program co-funded by the Agency for Management of University and Research Grants of the Government of Catalonia and by the COFUND program of the Marie Sklodowska-Curie actions under the framework program Horizon 2020 of the European Commission. JM gratefully acknowledges the financial support by ICREA under the ICREA Academia programme. JV acknowledges funding from the European Union's Horizon 2020 research and innovation programme under the Marie Sklodowska-Curie Grant agreement no 700525, 'NIOBE' and support from SEJI/2018/033 (Generalitat Valenciana). The work of AC is supported by a Professorship Grant (PP00P2_176884) of the Swiss National Science Foundation.

Data Availability Statement This manuscript has no associated data or the data will not be deposited. [Authors' comment: The experimental data used in the analysis presented in this paper has already been published by the different experiments cited in the text. Any further data related to the specifics of our analysis can be reproduced by following the procedures detailed in the article and predecessors, that we cite whenever it is appropriate.]

Open Access This article is distributed under the terms of the Creative Commons Attribution 4.0 International License (http://creativecomm ons.org/licenses/by/4.0/), which permits unrestricted use, distribution, and reproduction in any medium, provided you give appropriate credit to the original author(s) and the source, provide a link to the Creative Commons license, and indicate if changes were made. Funded by $\mathrm{SCOAP}^{3}$.

\section{Appendix A Correlations among fit parameters}

In addition to the confidence regions provided for the various scenarios in this article, we display here the correlation matrices for the most interesting NP scenarios. 


\section{Correlation matrices of fits to LFUV NP}

First, we present the correlations between fit parameters of the NP scenarios defined in Tables 2 and 3. These are all NP solutions whose parameters assess LFUV NP.

By order of appearance in Table 2, the correlations between the coefficients of all 2D scenarios with Pull $_{\mathrm{SM}} \gtrsim$ $5.3 \sigma$ are,

$$
\begin{aligned}
& \operatorname{Corr}\left(\mathcal{C}_{9 \mu}^{\mathrm{NP}}, \mathcal{C}_{10 \mu}^{\mathrm{NP}}\right)=\left(\begin{array}{cc}
1.00 & 0.30 \\
0.30 & 1.00
\end{array}\right) \\
& \operatorname{Corr}\left(\mathcal{C}_{9 \mu}^{\mathrm{NP}}, \mathcal{C}_{9^{\prime} \mu}\right)=\left(\begin{array}{cc}
1.00 & -0.39 \\
-0.39 & 1.00
\end{array}\right) \\
& \operatorname{Corr}\left(\mathcal{C}_{9 \mu}^{\mathrm{NP}}, \mathcal{C}_{10^{\prime} \mu}\right)=\left(\begin{array}{ll}
1.00 & 0.33 \\
0.33 & 1.00
\end{array}\right) \\
& \operatorname{Corr}\left(\mathcal{C}_{9 \mu}^{\mathrm{NP}}, \mathcal{C}_{9 e}^{\mathrm{NP}}\right)=\left(\begin{array}{ll}
1.00 & 0.51 \\
0.51 & 1.00
\end{array}\right) \\
& \operatorname{Corr}\left(\mathcal{C}_{9 \mu}^{\mathrm{NP}}=-\mathcal{C}_{9^{\prime} \mu}, \mathcal{C}_{10 \mu}^{\mathrm{NP}}=\mathcal{C}_{10^{\prime} \mu}\right)=\left(\begin{array}{cc}
1.00 & -0.17 \\
-0.17 & 1.00
\end{array}\right) \\
& \operatorname{Corr}\left(\mathcal{C}_{9 \mu}^{\mathrm{NP}}, \mathcal{C}_{9^{\prime} \mu}=-\mathcal{C}_{10^{\prime} \mu}\right)=\left(\begin{array}{cc}
1.00 & -0.34 \\
-0.34 & 1.00
\end{array}\right)
\end{aligned}
$$

The last two matrices correspond to Hyp. 1 and Hyp. 5 as defined in Table 2. Despite the high Pull SM $_{\text {of }}$ the 2D scenario $\left\{\mathcal{C}_{9 \mu}^{\mathrm{NP}}, \mathcal{C}_{7^{\prime}}\right\}(5.4 \sigma)$, its correlation matrix is not collected here due to the value of $\mathcal{C}_{7}$, being negligible, with tiny errors.

Regarding the 6D fit of Table 3 ,

$$
\text { Corr }_{6 \mathrm{D}}=\left(\begin{array}{cccccc}
1.00 & -0.34 & -0.07 & 0.06 & 0.02 & -0.03 \\
-0.34 & 1.00 & 0.24 & -0.06 & 0.04 & 0.24 \\
-0.07 & 0.24 & 1.00 & -0.13 & 0.61 & 0.59 \\
0.06 & -0.06 & -0.13 & 1.00 & -0.13 & -0.08 \\
0.02 & 0.04 & 0.61 & -0.13 & 1.00 & 0.85 \\
-0.03 & 0.24 & 0.59 & -0.08 & 0.85 & 1.00
\end{array}\right)
$$

where the columns are ordered as $\left\{\mathcal{C}_{7}^{\mathrm{NP}}\right.$, $\left.\mathcal{C}_{9 \mu}^{\mathrm{NP}}, \mathcal{C}_{10 \mu}^{\mathrm{NP}}, \mathcal{C}_{7^{\prime}}, \mathcal{C}_{9^{\prime} \mu}, \mathcal{C}_{10^{\prime} \mu}\right\}$

Interesting information can be extracted from Corr $6 \mathrm{D}$. Most of the coefficients do not show particularly strong correlations with the others except for the pairs $\left\{\mathcal{C}_{10 \mu}^{\mathrm{NP}}, \mathcal{C}_{9^{\prime} \mu}\right\}$, $\left\{\mathcal{C}_{10 \mu}^{\mathrm{NP}}, \mathcal{C}_{10^{\prime} \mu}\right\}$ and $\left\{\mathcal{C}_{9^{\prime} \mu}, \mathcal{C}_{10^{\prime} \mu}\right\}$, being the latter the highest in correlation. While $\mathcal{C}_{9 \mu}^{\mathrm{NP}}$ and $\mathcal{C}_{9^{\prime} \mu}$ show a non-negligible correlation in the fit to these coefficients only, in the $6 \mathrm{D}$ fit the aforementioned parameters are uncorrelated to a large extent. On the contrary, the correlation between $\mathcal{C}_{9 \mu}^{\mathrm{NP}}$ and $\mathcal{C}_{10 \mu}^{\mathrm{NP}}$ is very similar for both the global 6D and the 2D fit to these parameters alone.

\section{Correlation matrices of fits to LFUV-LFU NP}

Second, the correlations between fit parameters of scenarios with both LFUV and LFU NP have also been considered.
Below one can find the correlation matrices of scenarios 511 , in that order.

$$
\begin{aligned}
& \operatorname{Corr}\left(\mathcal{C}_{9 \mu}^{\mathrm{V}}, \mathcal{C}_{9}^{\mathrm{U}}=\mathcal{C}_{10}^{\mathrm{U}}, \mathcal{C}_{10 \mu}^{\mathrm{V}}\right)=\left(\begin{array}{ccc}
1.00 & -0.93 & 0.91 \\
-0.93 & 1.00 & -0.94 \\
0.91 & -0.94 & 1.00
\end{array}\right) \\
& \operatorname{Corr}\left(\mathcal{C}_{9 \mu}^{\mathrm{V}}=-\mathcal{C}_{10 \mu}^{\mathrm{V}}, \mathcal{C}_{9}^{\mathrm{U}}=\mathcal{C}_{10}^{\mathrm{U}}\right)=\left(\begin{array}{ll}
1.00 & 0.17 \\
0.17 & 1.00
\end{array}\right) \\
& \operatorname{Corr}\left(\mathcal{C}_{9 \mu}^{\mathrm{V}}, \mathcal{C}_{9}^{\mathrm{U}}\right)=\left(\begin{array}{cc}
1.00 & -0.85 \\
-0.85 & 1.00
\end{array}\right) \\
& \operatorname{Corr}\left(\mathcal{C}_{9 \mu}^{\mathrm{V}}=-\mathcal{C}_{10 \mu}^{\mathrm{V}}, \mathcal{C}_{9}^{\mathrm{U}}\right)=\left(\begin{array}{cc}
1.00 & -0.44 \\
-0.44 & 1.00
\end{array}\right) \\
& \operatorname{Corr}\left(\mathcal{C}_{9 \mu}^{\mathrm{V}}=-\mathcal{C}_{10 \mu}^{\mathrm{V}}, \mathcal{C}_{10}^{\mathrm{U}}\right)=\left(\begin{array}{cc}
1.00 & 0.69 \\
0.69 & 1.00
\end{array}\right) \\
& \operatorname{Corr}\left(\mathcal{C}_{9 \mu}^{\mathrm{V}}, \mathcal{C}_{10}^{\mathrm{U}}\right)=\left(\begin{array}{ll}
1.00 & 0.05 \\
0.05 & 1.00
\end{array}\right) \\
& \operatorname{Corr}\left(\mathcal{C}_{9 \mu}^{\mathrm{V}}, \mathcal{C}_{10^{\prime}}^{\mathrm{U}}\right)=\left(\begin{array}{ll}
1.00 & 0.20 \\
0.20 & 1.00
\end{array}\right)
\end{aligned}
$$

No significant changes can be observed when comparing with the results in App. 2 of Ref. [2]. As expected, $\mathcal{C}_{9 \mu}^{\mathrm{V}}$ and $\mathcal{C}_{9}^{\mathrm{U}}$ are highly anti-correlated, with its nominal value somewhat smaller than in [2]. Fit estimates of the parameters in scenario $\left\{\mathcal{C}_{9 \mu}^{\mathrm{V}}=-\mathcal{C}_{10 \mu}^{\mathrm{V}}, \mathcal{C}_{9}^{\mathrm{U}}=\mathcal{C}_{10}^{\mathrm{U}}\right\}$ are now slightly correlated, while before their correlation was negligible. Interestingly, however, we find the parameters of the new scenario $\left\{\mathcal{C}_{9 \mu}^{\mathrm{V}}, \mathcal{C}_{10}^{\mathrm{U}}\right\}$ statistically independent up to a large extent.

\section{References}

1. B. Capdevila, A. Crivellin, S. Descotes-Genon, J. Matias, J. Virto, JHEP 1801, 093 (2018). arXiv:1704.05340 [hep-ph]

2. M. Algueró, B. Capdevila, S. Descotes-Genon, P. Masjuan, J. Matias, Phys. Rev. D 99(7), 075017 (2019). arXiv:1809.08447 [hep-ph]

3. S. Descotes-Genon, L. Hofer, J. Matias, J. Virto, JHEP 1606, 092 (2016). arXiv: 1510.04239 [hep-ph]

4. S. Descotes-Genon, J. Matias, M. Ramon, J. Virto, JHEP 1301, 048 (2013). arXiv:1207.2753 [hep-ph]

5. B. Capdevila, S. Descotes-Genon, J. Matias, J. Virto, JHEP 1610, 075 (2016). arXiv: 1605.03156 [hep-ph]

6. R. Aaij et al., [LHCb Collaboration]. arXiv:1903.09252 [hep-ex]

7. S. Choudhury for the Belle collaboration, Measurement of Lepton Flavour Universality in $B$ decays at Belle, Talk at 'EPS-HEP Conference 2019'

8. R. Aaij et al., LHCb Collaboration, JHEP 1406, 133 (2014). arXiv: 1403.8044 [hep-ex]

9. A. Abdesselam et al., [Belle Collaboration]. arXiv:1904.02440 [hep-ex]

10. M. Aaboud et al., [ATLAS Collaboration]. arXiv:1812.03017 [hepex]

11. S. Aoki et al., [Flavour Lattice Averaging Group]. arXiv:1902.08191 [hep-lat]

12. S. Wehle et al., [Belle Collaboration], Phys. Rev. Lett. 118(11), 111801 (2017) arXiv:1612.05014 [hep-ex] 
13. A. Abdesselam et al., [Belle Collaboration]. arXiv:1604.04042 [hep-ex]

14. M. Ciuchini, A.M. Coutinho, M. Fedele, E. Franco, A. Paul, L. Silvestrini, M. Valli. arXiv:1903.09632 [hep-ph]

15. M. Algueró, B. Capdevila, S. Descotes-Genon, P. Masjuan, J. Matias. arXiv:1902.04900 [hep-ph]

16. A. Celis, J. Fuentes-Martin, A. Vicente, J. Virto, Phys. Rev. D 96(3), 035026 (2017). arXiv:1704.05672 [hep-ph]

17. W. Altmannshofer, S. Gori, M. Pospelov, I. Yavin, Phys. Rev. D 89, 095033 (2014). arXiv:1403.1269 [hep-ph]

18. A. Crivellin, G. D’Ambrosio, J. Heeck, Phys. Rev. Lett. 114, 151801 (2015). arXiv:1501.00993 [hep-ph]

19. A. Crivellin, C. Greub, D. Müller, F. Saturnino, Phys. Rev. Lett. 122(1), 011805 (2019). arXiv:1807.02068 [hep-ph]

20. A. Crivellin, D. Muller, C. Wiegand. arXiv:1903.10440 [hep-ph]

21. C. Bobeth, A.J. Buras, A. Celis, M. Jung, JHEP 1704, 079 (2017). arXiv:1609.04783 [hep-ph]

22. Y. Amhis et al., [HFLAV Collaboration], Eur. Phys. J. C 77(12), 895 (2017). arXiv:1612.07233 [hep-ex]

23. B. Grzadkowski, M. Iskrzynski, M. Misiak, J. Rosiek, JHEP 1010, 085 (2010). arXiv:1008.4884 [hep-ph]

24. B. Capdevila, A. Crivellin, S. Descotes-Genon, L. Hofer, J. Matias, Phys. Rev. Lett. 120(18), 181802 (2018). arXiv:1712.01919 [hep$\mathrm{ph}]$

25. R. Alonso, B. Grinstein, J. Martin Camalich, JHEP 1510, 184 (2015). arXiv:1505.05164 [hep-ph]
26. A. Greljo, G. Isidori, D. Marzocca, JHEP 1507, 142 (2015). arXiv:1506.01705 [hep-ph]

27. L. Calibbi, A. Crivellin, T. Ota, Phys. Rev. Lett. 115, 181801 (2015). arXiv:1506.02661 [hep-ph]

28. J. Aebischer, W. Altmannshofer, D. Guadagnoli, M. Reboud, P. Stangl, D.M. Straub. arXiv:1903.10434 [hep-ph]

29. M. Blanke, A. Crivellin, Phys. Rev. Lett. 121(1), 011801 (2018). arXiv:1801.07256 [hep-ph]

30. L. Di Luzio, A. Greljo, M. Nardecchia, Phys. Rev. D 96(11), 115011 (2017). arXiv:1708.08450 [hep-ph]

31. L. Calibbi, A. Crivellin, T. Li, Phys. Rev. D 98(11), 115002 (2018). arXiv:1709.00692 [hep-ph]

32. M. Bordone, C. Cornella, J. Fuentes-Martín, G. Isidori, Phys. Lett. B 779, 317 (2018). arXiv:1712.01368 [hep-ph]

33. A. Abdesselam et al., [Belle Collaboration]. arXiv:1904.08794 [hep-ex]

34. F. Kruger, J. Matias, Phys. Rev. D 71, 094009 (2005). arXiv:hep-ph/0502060

35. J. Matias, F. Mescia, M. Ramon, J. Virto, JHEP 1204, 104 (2012). arXiv:1202.4266 [hep-ph]

36. A.K. Alok, A. Dighe, S. Gangal, D. Kumar. arXiv:1903.09617 [hep-ph]

37. A. Arbey, T. Hurth, F. Mahmoudi, D.M. Santos, S. Neshatpour. arXiv: 1904.08399 [hep-ph]

38. A. Datta, J. Kumar, D. London. arXiv:1903.10086 [hep-ph] 